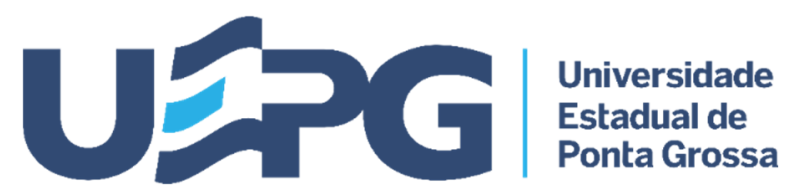

Revista Teias do Conhecimento

Ano 1, Numero 1, 2021

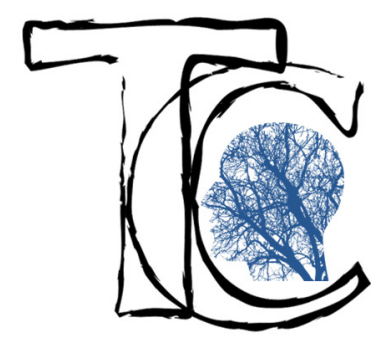

ISSN 2763-6739
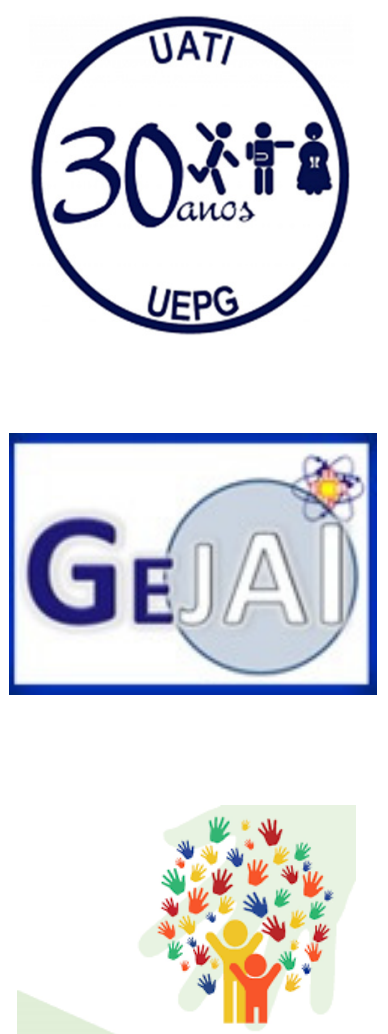

MESTRADO

EM EDUCAÇÃO INCLUSIVA

\title{
CORONAVIRUS: DESARROLLO SUSTENTABLE VERSUS ENCIERRO PANDÉMICO. REFLEXIONES SOBRE EL ADULTO MAYOR URBANO Y EL ADULTO MAYOR RURAL
}

\author{
http://doi.org/10.5212/RevTeiasConhecimento.v111.20210060
}

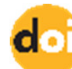

Erika Carcaño*

https://orcid.org/0000-0002-4097-0891

Alejandro Klein**

https://orcid.org/0000-0001-8216-345X

http://lattes.cnpq.br/3075254683116120

RESUMEN: En este trabajo proponemos algunas reflexiones sobre las repercusiones sociales y políticas que ha traído consigo el fenómeno de la pandemia de la coronavirus. Nos enfocamos especialmente en el grupo de los adultos mayores, sindicados desde el principio de la pandemia como especial grupo de riesgo. Pero observamos que la situación de los adultos mayores urbanos es diferente a la de los adultos mayores rurales. Estos últimos no han pasado por políticas de confinamiento ni segregación, probablemente en relación a entornos de desarrollo sustentable comunitario que neutralizan la "visión" de la naturaleza en términos de peligros, paranoicos o "ataques" virósicos, propugnando una visión armónica y ancestral fuertemente legitimada.

PALABRAS CLAVE: adultos mayores; coronavirus; desarrollo sustentable.

Doutora pela Universidad Autónoma Metropolitana (México) e Professora da Universidad de Guanajuato (México), 鼻 erikacarcano@gmail.com

** Pós-doutor pela Pontífica Universidade Católica do Rio de Janeiro (PUC/RIO) e Professor da Universidad de Guanajuato (México), 画 alejandroklein@hotmail.com 


\title{
CORONAVIRUS: SUSTAINABLE DEVELOPMENT VERSUS PANDEMIC ENCLOSURE. REFLECTIONS ON THE URBAN OLDER ADULT AND THE RURAL OLDER ADULT
}

\begin{abstract}
In this paper we propose some reflections on the social and political repercussions that the phenomenon of the coronavirus pandemic has brought with it. We especially focus on the group of older adults, identified since the beginning of the pandemic as a special risk group. But we observe that the situation of urban older adults is different from that of rural older adults. The latter have not gone through policies of confinement or segregation, probably in relation to environments of sustainable community development that neutralize the "vision" of nature in terms of dangers, paranoid or viral "attacks", advocating a harmonious and ancestral vision strongly legitimized.
\end{abstract}

KEY WORDS: older adults; coronavirus; sustainable development.

\section{CORONAVIRUS: DESENVOLVIMENTO SUSTENTÁVEL VERSUS TRAVAMENTO PANDÊMICO. REFLEXÕES SOBRE O ADULTO IDOSO URBANO E O ADULTO RURAL MAIS VELHO}

RESUMO: Neste artigo propomos algumas reflexões sobre as repercussões sociais e políticas que o fenômeno da pandemia do coronavírus trouxe consigo. Focamos especialmente o grupo de idosos, identificados desde o início da pandemia como um grupo de risco especial. Mas observamos que a situação dos idosos urbanos é diferente da dos idosos rurais. Estes últimos não passaram por políticas de confinamento ou segregação, provavelmente em relação a ambientes de desenvolvimento comunitário sustentável que neutralizem a "visão" da natureza em termos de perigos, "ataques" paranóicos ou virais, defendendo uma visão harmoniosa e ancestral fortemente legitimada.

PALAVRAS-CHAVE: idosos; coronavírus; desenvolvimento sustentável. 
Coronavirus: desarrollo sustentable versus encierro pandémico. reflexiones sobre el adulto mayor urbano y el adulto mayor rural

Erika Carcaño e Alejandro Klein

\section{INTRODUCCIÓN}

El objetivo de este escrito está vinculado al análisis de las repercusiones sociopolíticas que ha tenido el coronavirus en relación especialmente al grupo etáreo de los adultos mayores. De esta manera se indica que son tiempos dilemáticos para los adultos mayores. Signados de repente como grupo de riesgo por excelencia frente a la pandemia por el coronavirus, son confinados, estigmatizados, envejecidos de forma repentina y sin derecho a réplica. Surge una nueva corriente de edadismo que parece no encontrar opositores.

Sin embargo percibimos que la situación no es homogénea. Este edadismo renovado es mayoritariamente urbano. Observamos que en el medio rural no se generado confinamiento ni escenas de miedo. Por el contrario, el adulto mayor ha mantenido su situación de dignidad.

De esta manera la exposición propuesta en este trabajo trata de dar cuenta de qué categoría o categorías analíticas pueden dar cuentas de estas situaciones tan disímiles. A partir del concepto de desarrollo sustentable comunitario se realizan conclusiones que permiten afirmar que a pesar de una situación estigmatizante confinante, hay elementos que permiten el rescate de una posibilidad emancipatoria que no se debe perder de vista.

\section{LOS CONTUNDENTES DATOS DE LA TRANSICIÓN DEMOGRÁFICA} AVANZADA

Se espera que hasta el año 2050, el 21.8\% de la población mundial será de adultos mayores (UN, 2008). En los estudios de los años 90'se estimaba que el grupo de individuos de 75 años y más constituían el grupo de adultos mayores de mayor crecimiento. (Lawhorn, 1996). Sin embargo las últimas investigaciones señalan que el grupo de tercera edad que mayor crece es el de los centenarios (Leeson, 2009; 2013; UN, 2008).

Los números de incremento de edad hacia el año 2050 son absolutamente contundentes. De la actualidad al año 2050, la población de 60 años pasará de 667 a 
Coronavirus: desarrollo sustentable versus encierro pandémico. reflexiones sobre el adulto mayor urbano y el adulto mayor rural

Erika Carcaño e Alejandro Klein

2008 millones, en porcentajes de $10.2 \%$ a $21.8 \%$ en el total de población en los países más desarrollados. Asimismo para el año 2050, la población de 80 años pasará de 87 a 395 millones de personas.

Esta transición implica además el cambio de correlación entre la población de niños y de adultos mayores. Para el año 2050 en las zonas más desarrolladas, la proporción de niños será de $15.4 \%$ comparada con $32.6 \%$ de adultos mayores. En las regiones menos desarrolladas, mientras que en el año 2005 la proporción de niños era de $31 \%$ y de $8 \%$ de adultos mayores, para el año 2050 la proporción de adultos mayores será de $20.2 \%$ y de niños será de $20.3 \%$. (UN, 2008).

Al mismo tiempo se observa que el crecimiento de la población continúa pero se va desacelerando. Mientras que del año 1950 al 1990 pasó de 2.5 billones de personas a 5.3 billones de personas, del año 2025 al 2050 pasará de 8.0 billones de personas a "sólo" 9.2 billones de personas. Estos datos hacen pensar que en los países más desarrolladas el crecimiento será negativo y tenderá a decrecer, por lo que su probable crecimiento sólo pueda ser por migración. En cambio, en los países no desarrollados, el crecimiento aún podría ser (no en todas las regiones, hay que aclarar) natural (Leeson, 2011, 2014, 2014로 2015; UN, 2018).

\section{COMO HA IMPACTADO EL CORONAVIRUS EN LOS ADULTOS MAYORES DE LAS CIUDADES}

En el plazo de unos pocos meses y dentro de la pandemia del coronavirus, los adultos mayores urbanos han sido sedentarizados y recluidos en sus hogares, pensiones, casas de salud. En las mismas están vegetando, tienen miedo o comprenden muy poco de lo que está pasando. Pero están ya indefectiblemente recluidos, por un tiempo que nadie puede establecer ni calcular.

El consenso social está instalado: el viejo se debe confinar. Los viejos, se afirma a rajatabla, son el grupo de riesgo por excelencia. Sin embargo, basta indagar críticamente la información y nos enteramos que también los adultos y los niños y los jóvenes se contagian. Que en definitiva toda la Humanidad es grupo de riesgo. Sin embargo, con parsimonia y dogmatismo se impone la idea y se convence a la 
Coronavirus: desarrollo sustentable versus encierro pandémico. reflexiones sobre el adulto mayor urbano y el adulto mayor rural

Erika Carcaño e Alejandro Klein

población de que los viejos sea como sea, se deben aislar, confinar. La ciudad entera se transforma en un encierro y la vista de un adulto mayor por la calle se considera un escándalo

En definitiva: ¿Es exagerado decir que el confinamiento obligatorio al que están sometidos los adultos mayores presagia otras situaciones igualmente preocupantes? La advertencia ha sido dada: no se atenderán a los adultos mayores en caso de colapso sanitario...

Para que este enunciado social sea afirmado y legitimado con esta aprobación veloz y unánime, es porque algo ha pasado previamente en el imaginario social y en la estructura social que atañe a los adultos mayores hoy, al menos a los adultos mayores urbanos, despojados de repente de sus derechos de ciudadanía, de cuestionamiento y réplica.

\section{SITUACIONES CADA VEZ MÁS PREOCUPANTES DE EDADISMO}

La bibliografía más detallada y las historias de vida que se recolectaban daban cuenta de un nuevo modelo de vejez, llamado ahora "adulto mayor" o sujetos de la "tercera edad", donde ya no había espera alguna de la muerte, sino una renovación de la promesa de nuevas oportunidades, nuevas perspectivas, nuevos estilos desafiantes de vida. No toda la población vieja participó de este nuevo clima cultural e identitario, pero sí la suficiente como para calificar de "rupturista" a este grupo (Neugarten y Neugarten, 1982, 1986; Bengtson, 2001; Eisenberg, 1988; FeresCarneiro, 2005; Fisher, 1983; Harper, 2003, 2004; Leeson, 2013; Neugarten y Weinstein, 1961; Wilcoxon, 1987; Klein, 2015).

Podría elegirse entre muchas variables para estudiar este grupo renovador, pero un punto emprendedor y audaz radica en la abolición de la asociación de la vejez con la muerte. El incremento en la expectativa de sobrevida lleva a que se pase de la experiencia de la muerte como inminente a desplazada (Klein, 2015). Este grupo rupturista proyectó delante de sí vida y no muerte. Espacios abiertos, experimentación, nomadismo, gusto por lo nuevo y por la novedad dentro de una renovación portentosa del "cuidado de si" foucaultiano (Foucault, 1984, 1988). 
Coronavirus: desarrollo sustentable versus encierro pandémico. reflexiones sobre el adulto mayor urbano y el adulto mayor rural

Erika Carcaño e Alejandro Klein

Y sin embargo, y casi sin advertirlo, las cosas han dado un giro radical a partir del coronavirus o quizás, tomándolo como excusa: los adultos mayores urbanos se han transformado otra vez en vejez decrépita, enferma, asediada por la enfermedad, la epidemia y la muerte. Es lo que se percibe en el escenario político del COVID-19: el adulto mayor comienza a ser tratado de vuelta como "viejo" y asociado a políticas de decrepitud. De esta manera, las políticas emanadas del COVID-19 son exitosas porque no se está pensando al adulto mayor como ciudadano que opta, sino como un ser en peligro, desde una imagen fatigada, decrépita, de riesgo..

Se retoman rápidamente los estereotipos del "viejo", definido desde una enfermedad progresiva responsable de una multitud de cambios deficitarios fisiológicos y anatómicos (Haber, 1986), por los cuales el anciano se transforma en un ser improductivo e inútil socialmente, que se aparta cada vez más de la vida y la sociedad, aislado y desconectado en su propio mundo solitario (Cumming y Henry, 1961; Cole ,1997; Bourdelais, 1993).

\section{EL ADULTO MAYOR SÚBITAMENTE EXPIATORIO}

De este adulto mayor súbitamente envejecido parece que se vuelve a esperar lo que un autor como Erikson (2000) preconizaba: el viejo debe aceptar la muerte con un sentido de integración y plenitud, aceptando la vida que se ha vivido y por ende la muerte que se tiene por "delante".

De esta manera, el viejo urbano pasa a ser el depositario del ominoso trabajo de la muerte, transformado en un ritual de expiación: si el viejo es la figura social que parece que mejor se asocia a la muerte proveniente de la pandemia se lo recluye, en la ambivalencia de protegerlo, pero también, de dejarlo indemne (Nisbet, 1996).

El trabajo de "encapsular" la muerte en torno al anciano, se transforma en un ritual, que sólo es comprensible desde una época, que más allá del coronavirus, se caracteriza por la marcha, la aceleración y el empuje de sucesos, que como crisis permanente, se vuelven incomprensibles, generadores de angustia y de desesperación(Missenard, 1991). 
Coronavirus: desarrollo sustentable versus encierro pandémico. reflexiones sobre el adulto mayor urbano y el adulto mayor rural

Erika Carcaño e Alejandro Klein

Esta conjunción de sucesos desgraciados y crisis irreversible, hace que lo impensable encuentre un tope, a partir de lo cual se hace urgente encontrar una explicación a lo que está sucediendo. Un depositario, alguien que se haga cargo. Un receptor de las locuras y ansiedades colectivas (Pichón-Riviere, 1981)

Asumir una responsabilidad colectiva es una forma de encontrarle sentido a las cosas inexplicables. Si no se puede, el responsable designado encarna formas de "expiación" para esta culpa, que debe ser redimida o neutralizada. Redención -que desde un orden sacro -opera también como la promesa de poder pasar de un estado social actual a otro que está perdido, pero no duelado. Es decir, que permanece (desde lo mágico) como opción de retorno (Durkheim, 1968).

En la medida que los adultos mayores son transformados de vuelta en viejos decrépitos a merced de la muerte, se los coloca en esta "situación de sometimiento", a manera de ofrenda. Ante un desamparo estructural en el que se quiebran los sostenes imaginarios y simbólicos que aseguraban pertenencia y protección, se incrementa esta situación de ambigüedad, por la cual aparentemente son protegidos, tanto como son desamparados y entregados la muerte en un intento de restaurar equilibrios sociales y emocionales (Mandet, 2000).

Estos viejos urbanos operan como emergente de aquello que se ha vuelto imposible de duelar y que se expresa a través de la temática de la escasez. Ya no hay salud para todos, ya no hay camas para todos, ya no hay asistencia para todos, ya no hay respiradores para todos. Desde la escasez alguien sobra o molesta. Los viejos son ahora un estorbo. O causa de amenaza (Klein, 2015).

\section{EL IMPACTO DEL DESARROLLO SUSTENTABLE COMUNITARIO}

A pesar de la inmensa cantidad de datos que parecían indicar un panorama preocupante y hasta catastrófico para la población adulto mayor rural, la realidad no es tan negativa ni se constata que se estigmatize al adulto mayor desde un lugar de pérdida, arrebatamiento y pauperización sostenida a partir del coronavirus (Carcaño, 2018). 
Coronavirus: desarrollo sustentable versus encierro pandémico. reflexiones sobre el adulto mayor urbano y el adulto mayor rural

Erika Carcaño e Alejandro Klein

Muchos trabajos indican por el contrario que el adulto mayor rural mantiene diversas actividades y estrategias productivas ante los embates del modelo sanitario pandémico, que tienen como antecedentes actividades de agenciamiento en sus comunidades (Toledo y Moguel, 1999; De Molina y Toledo, 2011; Leff, 2004).

Asimismo, la consolidación de actividades productivas por parte de estas comunidades indígenas campesinas, les habilitan a una distribución colectiva del excedente que les permite junto a sus actividades políticas y sociales llevar a cabo estrategias para la defensa del territorio, donde la participación de los adultos mayores es también altamente relevante (Barkin y Rosas 2006; Barkin, et al, 2019).

El abordaje de la naturaleza que surge en estas comunidades rurales da cuenta de la complejidad de una cosmovisión basada en estructuras cognitivas, emocionales y comunitarias que se entrelazan entre sí de forma armónica e integrada (Carcaño, 2013; Carcaño y Klein, 2017). Las actividades que desarrollan en el espacio rural se dan alrededor de prácticas productivas organizadas bajo una gama de conocimientos tradicionales que relacionan su cosmovisión sobre la naturaleza y su sistema simbólico y de creencias (Toledo, 1993).

Podríamos pensar que esta cosmovisión integrada, ancestral y siempre renovada impide la visión de la naturaleza como virósica, pandémica o peligrosa, lo que neutraliza (o al menos minimiza) la ideología sanitaria persecutoria en torno al coronavirus propia del medio urbano, preservando inesperadamente el lugar social valorizado del adulto mayor rural, como sostén de estas tradiciones milenarias (Toledo y Barrera-Bassols, 2008).

Muchos de los procesos de conservación de esta tradición que se podría denominar "sagrada" sirve como base para comprender las estrategias que estas comunidades han generado como respuesta a los procesos de exclusión que han vivido históricamente y que con el paso del tiempo se han agudizado debido a la lógica de acumulación de capital desde contextos neoliberales.

De esta manera se podría decir que una de las contribuciones decisivas que realiza la tercera edad rural es la forma en la cual las comunidades indígenas 
Coronavirus: desarrollo sustentable versus encierro pandémico. reflexiones sobre el adulto mayor urbano y el adulto mayor rural

Erika Carcaño e Alejandro Klein

campesinas están haciendo frente a los retos impuestos por el modelo extractivista. Los adultos mayores del medio rural pasan a ser depositarios, custodios y garantes de un modelo de desarrollo sustentable alternativo, que es a su vez base de una identidad digna y reconocida del adulto mayor rural (; Beaucage, 2017).

\section{TRADICIÓN Y RENOVACIÓN DEL ADULTO MAYOR RURAL}

Estas comunidades indígenas campesinas y sus miembros de la tercera edad no solo hacen frente a las políticas neoliberales de exclusión y pauperización, sino que generan, además, de forma creativa y contundente, alternativas coherentes que se nutren de posicionamientos resilientes, sociales, culturales y económicos, de enorme importancia y que aún no han sido suficientemente estudiados.

Consideramos que estas situaciones implican un entrelazamiento entre prácticas de solidaridad e investimientos sociales fraternizantes en forma de empoderamiento. La capacidad empoderante apunta a una posibilidad de historización colectiva, de construcción de proyectos y porvenir entre muchos donde al mismo tiempo, se sostienen fundamentos comunitarios y rurales imprescindibles que renuevan pactos sociales ancestrales (Zukerfeld, 2003; Carcaño, 2018).

En los grupos indígenas mesoamericanos esta posibilidad se amplifica en el grupo de adultos mayores. Erróneamente, se entiende estereotipadamente que los mismos son conservadores, pero la evidencia recogida apunta justamente a lo contrario (Klein, 2015). Son grupos indígenas rurales capaces de ruptura, cambio y movilización actualizando dispositivos fraternos y solidarios, actuando como custodios de una tradición instituyente que enlaza tradición a capacidad emancipatoria (Klein, 2018; Vázquez y Velázquez, 1998; Vázquez, 2002).

Sin duda, desde estas nuevas realidades sociales y rurales la palabra del viejo se mantiene aún como la palabra de lo sagrado. Pero este lugar de lo sagrado y lo transmisible recibe en estas sociedades una reformulación que no puede sino alcanzar a un redimensionamiento social y cultural, que posibilita un tiempo que desde lo ancestral se proyecta hacia un "futuro" histórico (Villoro, 2003; Behera, 2006). 
Coronavirus: desarrollo sustentable versus encierro pandémico. reflexiones sobre el adulto mayor urbano y el adulto mayor rural

Erika Carcaño e Alejandro Klein

Podemos pensar que el agenciamiento comunitario que llevan a cabo algunas comunidades indígenas campesinas se caracteriza antes que nada por profundos procesos económicos, sociales y culturales que no tienen por qué entrar en contradicción con herencias tradicionales y que de ninguna manera queda paralizada ante un pasado ancestral.

Esta posibilidad de alternativas de gestión social y económica, con prioridad de los procesos de autonomía y resiliencia, se encuentran cada vez más presentes en muchas organizaciones comunitarias indígenas, rompiendo, como ya lo señalamos, con viejos paradigmas, e imponiendo la necesidad de explorar nuevos modelos explicativos frente a procesos cada vez más decisivos que atañen al entrelazamiento entre procesos económicos, sociales y culturales.

Por lo pronto indicamos que la tradición legitimada de un desarrollo sustentable marca una estabilidad en el tejido social de los adultos mayores rurales, que marca una radical diferencia con los adultos mayores urbanos, más dependientes de ciclos de inestabilidad social y cultural que los terminan perjudicando, como ya indicamos en el caso del coronavirus. 
Coronavirus: desarrollo sustentable versus encierro pandémico. reflexiones sobre el adulto mayor urbano y el adulto mayor rural

Erika Carcaño e Alejandro Klein

\section{CONCLUSIONES}

Aunque son hipótesis a desarrollar parece ser que en tiempos donde se enarbola y protege la estabilidad y el resguardo social, los adultos mayores tienen posibilidades sociales y culturales de dignidad y protección. Tiempos significativos donde el adulto mayor es capaz de constituirse en sujeto emancipado y en pleno proceso de empoderamiento.

Por el contrario en tiempos de enorme estabilidad, donde una pandemia rápidamente establece un del: "sálvese quién pueda", los adultos mayores quedan expuestos, desamparados, azorados, transformados nuevamente en viejos decrépitos a solas y arrinconados con la muerte, en una práctica expiatoria y preocupante. Punto especialmente claro en el caso de los adultos mayores urbanos.

De esta manera, los viejos no mueren sólo por el coronavirus, mueren también por la falta de asistencia deliberada y asistida. Lo que se llama: el colapso del sistema sanitario. En este sentido hay que tener en cuenta que el coronavirus no es solo un problema virósico y sanitario. Las decisiones y no decisiones que se toman frente a él, así como las estrategias, retrasos, prioridades, y distribuciones que conllevan, lo transforman en un dispositivo social y político.

Sin embargo, otra parece ser la situación de los adultos mayores a nivel rural. El agenciamiento comunitario que los mismos ponen de manifiesto desemboca en prácticas sustentables que impactan en el bienestar comunitario, en una mejor calidad de vida, y en una preservación de prácticas identitarias que aunque renovadas, son milenarias y remiten a un estado de dignidad legitimado y capaz de soportar los embates culturales de la escasez, la expiación y la política del chivo expiatorio, encarnados en una visión paranoica de la naturaleza y por ende, a una visión "virósica" que antecede y explica culturalmente una pandemia que no es sólo biológica.

Consideramos importante entonces tener en cuenta estos escenarios económicos y culturales alternativos donde las comunidades indígenas más que "combatir" a la naturaleza, se encuentran en una posición instituyente de "diálogo" permanente con la "Madre-Tierra", los que los perfila hacia formas inéditas y 
Coronavirus: desarrollo sustentable versus encierro pandémico. reflexiones sobre el adulto mayor urbano y el adulto mayor rural

Erika Carcaño e Alejandro Klein

novedosas de articulación económica, cultural y ambiental y los preserva de climas de confinamiento y muerte.

Los adultos mayores rurales ya no son simplemente las "autoridades" políticas de su comunidad o los depositarios de una memoria ancestral. Su credibilidad ya no está garantizada sólo por la tradición, sino además por la sorprendente capacidad de cambio y experimentación subjetiva que demuestran, posicionándose en lugares claves de la cotidianeidad cultural, económica y social de sus comunidades, lo que sobrepasa en mucho el lugar de vulnerabilidad que el adulto mayor urbano desafortunadamente revela.. 
Coronavirus: desarrollo sustentable versus encierro pandémico. reflexiones sobre el adulto mayor urbano y el adulto mayor rural

Erika Carcaño e Alejandro Klein

\section{REFERÊNCIAS}

Barkin, D., y Rosas, M. (2006). ¿Es posible un modelo alterno de acumulación?. Polis. Revista de la Universidad Bolivariana, 5 (13), 361-371.

Barkin, D. y cols. (2019). Sujeto Revolucionario desde la comunidad y sus modalidades de transformación social. Revista Cultura y Representaciones Sociales, 14 (27), 35-77.

Beaucage, P.(2007). Los pueblos indios y campesinos en su lucha por la defensa y el aprovechamiento de los recursos naturales en América Latina: Sistematización de experiencias recientes. Extraído el 12 de julio de 2017 desde:

http://es.scribd.com/doc/70124716/Los-Pueblos-Indios-Campesinos

Behera, M. (2006). Globalising. Rural Development. Competing Paradigms and Emergining Realities. Londres: Sage Publications.

Bengtson, V. L. (2001). Beyond the nuclear family: The increasing importance of multigenerational relationships en American society. Journal of Marriage and the Family, 63, 1-16.

Bourdelais, P. (1993). L’Âge de la vieillesse. Histoire du vieillissement de la population.

Paris : Odile Jacob.

Carcaño, E. (2013). Las mujeres indígenas en la Nueva Ruralidad Comunitaria (NRC) y su implicación en la generación de excedentes. El caso de la organización

"Masehualsihuamej Monsenyolchicahuanij". Tesis Doctoral. Ciudad de México: Universidad Autónoma Metropolitana.

Carcaño, E. (2018). Gerontoruralidad Alternativa: Otra mirada sobre la vejez en el espacio rural. Larna Newsletter, 8, Spanish version.

Carcaño, E. y Klein, A. (2017). La vejez problematizada. Imaginarios sociales que toleran lo que otrora era intolerable. Revista de Ciências Humanas, 51, (2), 477-493.

Cole, T. (1997). The Journey of Life. Cambridge: University Press, MA. Cumming, E. y William, H. (1961). Growing Old. The Process of Disengagement. Nueva York: Basic Books.

De Molina, M. y Toledo V. (2011). Metabolismos, naturaleza e historia. Hacia una teoría de las transformaciones socioecológicas. Barcelona: Ed. Icaria.

Durkheim, É. (1968). Las formas elementales de la vida religiosa. Buenos Aires:

Editorial Schapire.

Eisenberg, G. (1988). Grandchildren's perspectives on relationships with

grandparents: the influence of gender across generations. Sex Roles, 19, 205-217.

Erikson, E. (2000). El ciclo vital completado. Madrid: Paidós Ibérica.

Feres-Carneiro, T. (2005). (Ed). Família e Casal. Efeitos da Contemporaneidade. Rio de Janeiro: Editora Puc-Rio.

Fisher, L.R. (1983). Transition to grand motherhood. International. Journal of Aging and Human Development, 16, (1), 67-78.

Foucault, M. (1984). Deux essais sur el sujet et le pouvoir. En Dreyfus, H. y Raibow, P. (Ed), Michel Foucault. Un parcours philosophique (pp. 201-334). Paris: Gallimard.

Foucault, M. (1988). La ética del cuidado de sí como práctica de libertad. En Foucault, M. Coleçao Ditos \& Escritos V. (pp 137-169). Brasilia: Política Forense Universitaria. 
Coronavirus: desarrollo sustentable versus encierro pandémico. reflexiones sobre el adulto mayor urbano y el adulto mayor rural Erika Carcaño e Alejandro Klein

Harper, S. (2003). Changing families as European societies. European Journal of Sociology, 44, (02), 155-184.

Harper, S. (2004). Families in Ageing Societies. A Multi-Disciplinary Approach. Oxford: Oxford University Press.

Klein, A. (2015). Del Anciano al Adulto mayor-Procesos psicosociales, de salud mental, familiares y generacionales. Ciudad de México: Plaza y Valdez Editores.

Klein, A. (2018). La vejez problematizada. Imaginarios sociales que toleran lo que otrora era intolerable. Desacatos, 57, 120-135.

Leeson, G. (2009). Demography, Politics and Policy in Europe. En Ludlow, Peter (Ed.) Setting EU Priorities 2009 Pp. 102-124). Ponte de Lima: The European Strategy Forum.

Leeson, G. (2013). The demographics of population ageing in Latin America, the Caribbean and the Iberian Peninsula, 1950-2050. En Montes de Oca, Verónica (Ed). La agenda del Envejecimiento y las Políticas Públicas Hoy (pp. 53-74). Ciudad de México: Instituto de Investigaciones Sociales.

Lawhorn, Th. (1996). Seniors adults and computers in the 1900's. Educational Gerontology, 22, (2), 193-201.

Leff, E. (2004). Racionalidad Ambiental. La reapropiación social de la naturaleza. Mexico: Siglo XXI.

Mandet, E. (2002). Inmolación y juventud. Symposium: civilización y barbarie: una visión psicoanalítica. Buenos Aires: Asociación Escuela Argentina de Psicoterapia para Graduados.

Missenard , A. (1991). Lo negativo. Figuras y modalidades. Buenos Aires: Amorrourtu.

Neugarten, B., y Weinstein, K. (1961). The chanching american grandparents. Journal of Marriage and Family, 26,(2), 199-204.

Neugarten, B. y Neugarten, D (1986). Age in the aging society. En Piíer, A. y Bronte, L. (Ed.). Our aging society: Promise and paradox. (pp. 123-132). Nueva York: W.W. Norton.

Neugarten, B. (1982). Psychological aspects of aging and illness. Clinical Issues in Geriatric Psychiatry, 25, (2), 123-131.

Nisbet, R. (1996). La formación del pensamiento sociológico. Vol. I y II. Buenos Aires: Amorrortu.

Pichon-Riviere, E. (1981). Del Psicoanálisis a la Psicología Social. Buenos Aires: Nueva Visión.

Toledo, V. y Moguel, P. (1999) Café, luchas indígenas y sostenibilidad; el caso de México. Extraído el 12 de mayo de 2016 desde:

http://biblioteca2012.hegoa.efaber.net/system/ebooks/8963/original/Cafe_luchas_indigenas _y_sostenibilidad.pdf

Toledo, V. y Barrera-Bassols, N. (2008). La memoria biocultural: La importancia ecológica de las sabidurías tradicionales. Barcelona: Ed. Icaria.

United Nations (2008). World Population prospects (2008 revision) Extraído el 14 de julio de 2017 desde: 
Coronavirus: desarrollo sustentable versus encierro pandémico. reflexiones sobre el adulto mayor urbano y el adulto mayor rural

Erika Carcaño e Alejandro Klein

http://www.un.org/esa/population/publications/wpp2008/wpp2008_highlights.pdf

United Nations (2018). World Urbanization Prospects (2018 Revision). Extraído el 2 de mayo de 2019 desde: https://population.un.org/wup/Publications/Files/WUP2018-Report.pdf

Vázquez, V. y Velázquez, M. (1998). Miradas al futuro. Hacia la construcción de sociedades sustentables con equidad de género. México: UNAM.

Vázquez, V. (2002). ¿Quién cosecha lo sembrado? Relaciones de género en un área natural protegida mexicana. México: Plaza y Valdez.

Villoro, L. (2003). De la libertad a la comunidad. México: Fondo de Cultura Económica.

Wilcoxon, S. (1987). Grandparents and grandchildren: an often neglected relationship between significant others. Journey of Counseling and Development, 65, 289-290.

Zukerfeld, R. (2003). Procesos terciarios, creación, resiliencia y prácticas sociales transformadoras. Extraído el 12 de junio de 2017 desde:

http://www.aperturas.org/14zukerfeldautorizado.html. 


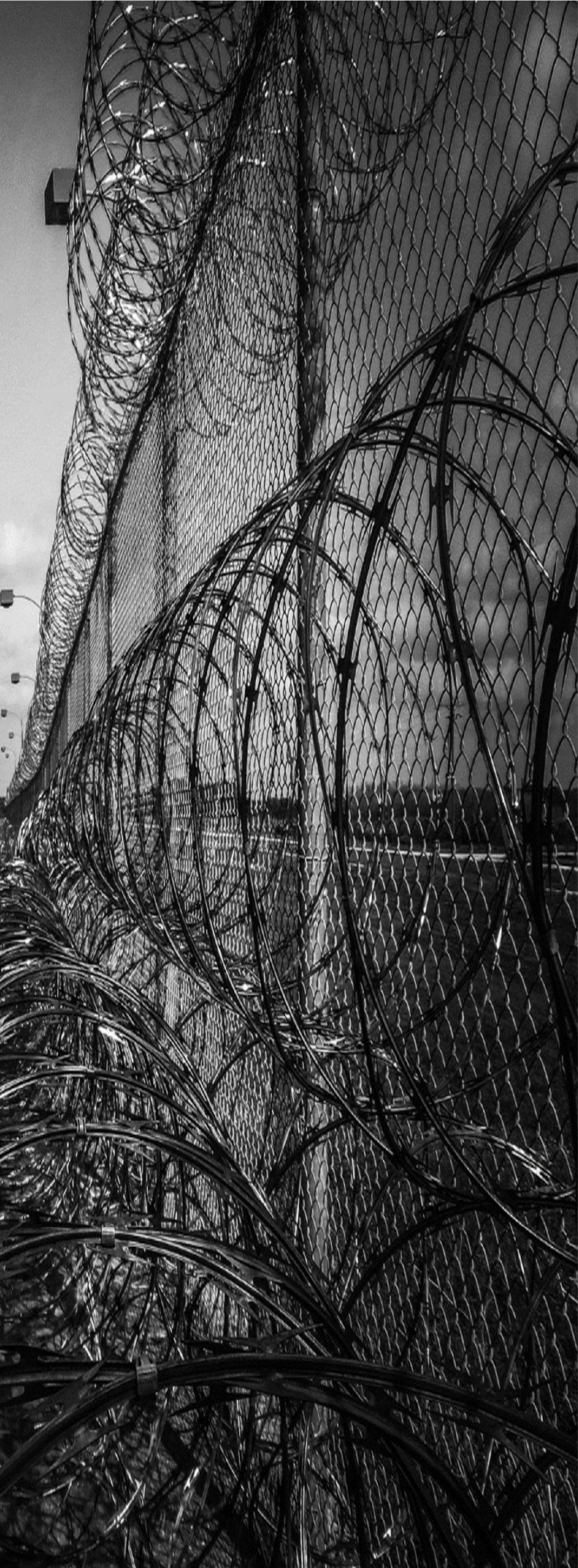

